\title{
Diagnósticos de enfermagem no perioperatório de cirurgia cardíaca*
}

\author{
NURSING DIAGNOSIS IN THE PERIOPERATIVE OF CARDIAC SURGERY
}

DIAGNÓSTICOS DE ENFERMERÍA EN EL PERIOPERATORIO DE CIRUGÍA CARDIACA

\author{
Luzia Elaine Galdeano', Lídia Aparecida Rossi², Cláudia B. dos Santos ${ }^{3}$, Rosana Aparecida S. Dantas ${ }^{4}$
}

\footnotetext{
* Estudo realizado com o apoio da Fundação de Amparo à

Pesquisa do Estado de São Paulo FAPESP. Extraído da dissertação de mestrado

"Diagnósticos de enfermagem de pacientes no período perioperatório de cirurgia cardíaca", Escola de Enfermagem de Ribeirão Preto da Universidade de São Paulo (EERP/USP),2002.

1 Mestre pela EERP/ USP. Professora do Departamento de Enfermagem MédicoCirúrgica da Faculdade de Enfermagem do Hospital Israelita Albert Einstein. legaldeano@hotmail.com

2 Professora Associada do Departamento de Enfermagem Geral e Especializada da EERP/USP.

3 Professora do Departamento Materno Infantil e de Saúde Pública da EERP/USP.

4 Professora Doutora do Departamento de Enfermagem Geral e Especializada da EERP/USP.
}

\section{RESUMO}

Foram identificados os diagnósticos de enfermagem de pacientes no período perioperatório de cirurgia cardíaca e verificada a concordância ou não entre a primeira autora e outros enfermeiros na identificação desses diagnósticos. Dezessete pacientes admitidos para cirurgia cardíaca foram avaliados utilizando-se instrumentos para o período perioperatório. No pré-operatório, das nove categorias diagnósticas identificadas pela primeira autora, três apresentaram concordância com os enfermeiros (exemplo: Intolerância à atividade). No transoperatório, sete categorias apresentaram concordância entre enfermeiros e primeira autora (exemplo: Risco para infecção). No pós-operatório imediato, 11 categorias apresentaram concordância entre enfermeiros e primeira autora (exemplo: Risco para disfunção neurovascular periférica).

\section{DESCRITORES}

Diagnóstico de enfermagem. Assistência perioperatória. Cirurgia torácica.

\section{ABSTRACT}

This study identified the nursing diagnoses of patients in the perioperative period of cardiac surgeries and verified the existence or not of agreement between the first author and other nurses in the identification of these diagnoses. Seventeen patients admitted for cardiac surgery were submitted to evaluation using instruments for the perioperative period. In the pre-operative, out of the 9 diagnosis categories identified by the first author, three agreed with the nurses (example: Activity intolerance). In the trans-operative, seven categories showed agreement among the nurses and the first author (example: Risk for infection). In the postoperative, 11 categories showed agreement among the nurses and the first author (example: Risk for peripheral neurovascular dysfunction).

\section{KEY WORDS}

Nursing diagnosis. Perioperative care. Thoracic surgery.

\section{RESUMEN}

Fueron identificados los diagnósticos de enfermería de pacientes en el periodo peri-operatorio de cirugía cardiaca y verificada la existencia o no de concordancia entre la primera investigadora y otros enfermeros en la identificación de esos diagnósticos. Diecisiete pacientes admitidos para cirugía cardiaca fueron sometidos a evaluación, utilizando un instrumento para cada momento del periodo peri operatorio. En el peri-operatorio, de los diagnósticos identificados por la primera investigadora, tres coincidieron con los enfermeros (ejemplo Intolerancia a la actividad). En el trans-operatorio, siete categorías coincidieron entre los enfermeros y la primera investigadora (ejemplo: Riesgo de infección). En el post-operatorio inmediato, 11 categorías coincidieron entre los enfermeros y la primera investigadora (ejemplo: Riesgo para disfunción neurovascular periférica).

\section{DESCRIPTORES}

Diagnostico de enfermería. Atención perioperativa. Cirugía torácica. 


\section{INTRODUÇÃO}

A morte súbita cardíaca é a manifestação letal mais comum da doença cardíaca. Achados clínicos e patológicos sugerem que paciente com doença arterial coronariana, cardiomiopatias, arritmias cardíacas ou doença cardíaca hipertensiva possuem altos riscos de morte súbita. Na maior parte dos casos os pacientes desenvolvem uma taquicardia ventricular que rapidamente progride para uma fibrilação ventricular e colapso circulatório ${ }^{(1)}$.

Um estudo realizado nos Estados Unidos concluiu que das 719.456 mortes de origem cardíaca entre adultos com idade superior a 35 anos no ano de 1998, $456.076(63,0 \%)$ foram definidas como morte súbita ${ }^{(2)}$.

O tratamento da doença cardíaca pode ser clínico ou cirúrgico, ambos com o objetivo de restabelecer a capacidade funcional do coração de forma a diminuir a sintomatologia e proporcionar ao indivíduo o retorno às suas atividades normais.

A cirurgia cardíaca é realizada quando a probabilidade de uma vida útil é maior com o tratamento cirúrgico que com o tratamento clínico. Existem três tipos de cirurgia cardíaca: as corretoras (fechamento de canal arterial, de defeito de septo atrial e ventricular), as reconstrutoras (revascula-rização do miocárdio, plastia de valva aórtica, mitral ou tricúspide) e as substitutivas (trocas valvares e transplantes) ${ }^{(3)}$.

Não foram encontrados estudos nacionais, com D.E. no perioperatório de cirurgia cardíaca, considerando-se pré, trans e pós-operatório to do risco para doença coronariana, alteração da função respiratória, alteração da circulação periférica, ansiedade, déficit de conhecimento e mobilidade física prejudicada ${ }^{(5)}$.

Não foram encontrados estudos nacionais que se reportam à identificação dos diagnósticos de enfermagem de pacientes que se encontram no período perioperatório de cirurgia cardíaca, considerando os três períodos (pré, trans e pós-operatórios).

Um outro estudo identificou e analisou os diagnósticos de enfermagem relacionados ao sistema respiratório de pacientes no período pré e pós-operatório de cirurgia cardíaca. Os diagnósticos de enfermagem, de origem respiratória, identificados pela autora foram: Troca de gases prejudicada (25\%); Eliminação traqueobrônquica ineficaz (22,2\%); Padrão respiratório ineficaz (38,3\%); Risco para troca de gases prejudicada $(7,9 \%)$ e Risco para padrão respiratório ineficaz $(6,6 \%)^{(6)}$.

A identificação dos diagnósticos de enfermagem de pacientes que se encontram no período perioperatório de cirurgia cardíaca poderá direcionar a assistência de enfermagem a esses pacientes, fornecendo subsídios para a elaboração do plano de cuidados individualizado, implementação de intervenções, treinamento e qualificação da equipe.

Na literatura, encontramos artigos que se reportam à identificação dos diagnósticos de enfermagem de pacientes com doença cardiovascular que se encontram em unida des de terapia intensiva ${ }^{(7-11)}$.
O tipo mais comum de cirurgia cardíaca reconstrutora é a revascularização do miocárdio. O objetivo dessa cirurgia é aliviar a angina e preservar a função do miocárdio. Estudos demonstram que $90 \%$ dos pacientes pós-revascularização demonstram melhora da função cardíaca, com redução da necessidade de utilização de betablo-queadores e nitratos e $60 \%$ apresentam eliminação de episódios anginosos, resultando em melhoria da qualidade de vida ${ }^{(4)}$.

A complexidade de cuidados requeridos por indivíduos com problemas cardíacos, cujas condições de saúde sofrem mudanças constantes, e que necessitam de intervenções de enfermagem imediatas, despertou o interesse em identificar os diagnósticos de enfermagem no período perioperatório de cirurgia cardíaca.

Em um estudo preliminar foram identificadas as características definidoras e as etiologias dos dez mais freqüentes diagnósticos de enfermagem, estabelecidos por enfermeiros especialistas, em 158 pacientes com doença cardiovascular. Os dez mais freqüentes diagnósticos de enfermagem identificados nesse estudo incluíam: alteração na circulação coronariana, alteração do conforto, diminuição débito cardíaco, diminuição da tolerância à atividade física, aumen-
Sabe-se que ocorrem, com muita frequiência, discordâncias entre diagnósticos identificados por diferentes enfermeiros quando examinam um mesmo indivíduo e até por um mesmo enfermeiro quando examina o mesmo indivíduo duas vezes. Os desacordos clínicos no estabelecimento de diagnósticos entre profissionais são comuns e podem estar relacionados a fatores inerentes ao examinador (habilidade, treinamento, conhecimento teórico), ao paciente (fisiopatologia, tratamento, variações biológicas) e ao exame ${ }^{(12-13)}$.

Em um encontro da Association of American Medical College Council of Deans, em 1975, foi construída uma lista com os principais erros no processo de raciocínio diagnóstico. Os erros no estabelecimento do diagnóstico foram classificados, nesta lista, em quatro categorias: omissão, quando dados clínicos importantes são ignorados; conclusão prematura, quando o diagnóstico identificado não está muito caracterizado pelos dados existentes; síntese errada, quando os dados disponíveis contradizem a conclusão, isto é, os dados relacionados contradizem a inferência diagnóstica e sintese inadequada, quando as conclusões são baseadas em dados clínicos não levantados ${ }^{(14)}$. 
Os objetivos desse estudo foram identificar os diagnósticos de enfermagem de pacientes no período perioperatório de cirurgia cardíaca e verificar a existência ou não de concordância entre a primeira autora do estudo, que avaliou os pacientes e identificou os diagnósticos de enfermagem e outros enfermeiros que tiveram acesso apenas aos dados coletados.

\section{MATERIAL E MÉTODO}

O estudo foi realizado no Hospital das Clínicas da Faculdade de Medicina de Ribeirão Preto da Universidade de São Paulo, no período de janeiro à maio de 2001. O grupo de estudo foi constituído de 17 pacientes adultos, independente do sexo, internados nesta instituição para serem submetidos à cirurgia cardíaca. Os critérios adotados para a inclusão no grupo foram: ser adulto, submetido à revascularização do miocárdio, troca ou plastia de valvas e demonstrar interesse na participação do estudo, com anuência por escrito, a partir da assinatura do Termo de Consentimento Livre Esclarecido.

Os instrumentos de coleta de dados foram elaborados com base no Modelo Conceitual de Wanda Horta, que se fundamenta nas necessidades humanas básicas propostas por Maslow ${ }^{(15)}$ e na classificação de Mohana ${ }^{(16)}$. Foram construídos três instrumentos para avaliação do paciente em cada um dos períodos perioperatório (pré, trans e pósoperatório imediato). Para construção dos instrumentos foi agrupado, para cada necessidade básica, o máximo possível de elementos que poderiam caracterizar os diagnósticos de enfermagem, tendo por base a literatura e a experiência profissional. Após a elaboração dos instrumentos, seis enfermeiras, com experiência em cardiologia e conhecimento em diagnóstico de enfermagem, realizaram a validação de aparência e conteúdo dos instrumentos. Foram sugeridas algumas alterações, em sua maioria, relacionadas à forma e apresentação dos instrumentos; as sugestões foram aceitas, aumentando a clareza e facilitando a leitura e compreensão dos itens.

Realizada a validação dos instrumentos, foi iniciado um pré-teste, mediante a aplicação dos instrumentos de coleta de dados em cinco pacientes, que atenderam ao critério de inclusão, com o objetivo de: identificar a melhor forma de aplicação dos instrumentos, verificar a adequação do conteúdo, identificar problemas que poderiam interferir na fidedignidade dos dados e avaliar o tempo gasto durante a coleta de dados.

Terminado o pré-teste e realizados os ajustes necessários, foi iniciada a coleta de dados. A coleta de dados foi realizada pela primeira autora do estudo (LEG) e obedeceu à maneira descrita a seguir: após consultar a escala cirúrgica (sistema de anotação das cirurgias eletivas e controle de leito/registro/paciente) e constatar que o paciente seria um provável integrante do grupo em estudo, foi realizada a visita à enfermaria para realização da primeira etapa da coleta (período pré-operatório). Após esclarecimentos sobre o estudo, o paciente foi indagado quanto ao desejo em participar e, mediante resposta positiva e consentimento por escrito, iniciou-se a entrevista e o exame físico. O segundo momento da coleta de dados foi realizado no centro cirúrgico, onde o mesmo paciente que concordou em participar da pesquisa (no período pré-operatório) foi avaliado novamente, durante a cirurgia. Para finalizar, o paciente foi avaliado nas primeiras seis horas do período pós-operatório, na Unidade de Recuperação Pós-Cirurgia Cardíaca.

Após cada coleta de dados, com cada paciente, foram realizadas a análise e síntese dos dados obtidos, utilizandose o processo raciocínio diagnóstico proposto por Risner ${ }^{(17)}$. Em seguida, foram estabelecidos os diagnósticos de enfermagem por LEG, com base na Taxonomia I da North American Nursing Diagnosis Association (Nanda) ${ }^{(18)}$.

É importante ressaltar que, embora as intervenções não fossem o foco de atenção deste estudo, os diagnósticos de enfermagem identificados foram comunicados ao enfermeiro responsável pela unidade, no intuito de proporcionar subsídios para a elaboração de um plano de cuidados específico para aqueles pacientes.

Os instrumentos de coleta de dados preenchidos por LEG foram entregues a três enfermeiros, que atuavam na área de cardiologia e possuíam conhecimento em diagnóstico de enfermagem, para que identificassem os diagnósticos de cada paciente, de forma individual, conforme seu julgamento, seguindo as etapas do processo de raciocínio diagnósticos proposto por Risner ${ }^{(17)}$ e a Taxonomia I da NANDA $^{(18)}$. O objetivo dessa etapa foi verificar se os profissionais identificariam ou não, os mesmos diagnósticos, a partir da análise dos dados coletados, de forma a validar ou não os diagnósticos identificados previamente por LEG. Os instrumentos de coleta, já preenchidos por LEG, foram entregues pessoalmente, em um encontro com os enfermeiros convidados, quando foram fornecidas as instruções quanto ao tipo de processo de raciocínio diagnóstico e à Taxonomia que deveriam ser utilizados para o estabelecimento dos diagnósticos.

Cada enfermeiro definiu, individualmente, os diagnósticos dos 17 sujeitos, nos três períodos. É importante ressaltar, que os diagnósticos de enfermagem identificados por LEG não foram revelados aos enfermeiros convidados para participar do estudo.

Antes de testar a concordância ou discordância entre os diagnósticos identificados pelos enfermeiros e por LEG, foi realizada uma avaliação geral das categorias identificadas somente pelos enfermeiros, mediante a aplicação dos testes estatísticos não paramétricos ${ }^{(19)}$. Para isso, foram agrupadas todas as categorias diagnósticas identificadas pelos 
enfermeiros em cada um dos períodos (pré, trans e pós-operatório imediato).

A aplicação do teste não paramétrico Friedman ${ }^{(19)}$ teve como objetivo avaliar a homogeneidade no que diz respeito a frequiência de cada categoria diagnóstica identificada pelos três enfermeiros convidados. Buscou-se com esse teste avaliar se os três enfermeiros identificariam freqüências semelhantes de diagnósticos e verificar se não existiriam tendências ou vícios, para que então, pudessem ser comparados os diagnósticos identificados por esses enfermeiros com os identificados por LEG. Dessa forma, foram atribuídos postos às freqüências de cada categoria diagnóstica identificada por cada enfermeiro.

No período pré-operatório, o teste estatístico ${ }^{(19)}$ não mostrou a existência de diferença estatisticamente significante entre os totais de postos para frequiência dos três enfermeiros $\left(\chi_{2 ; 0,0}^{2}=2,83 ; p=0,24\right)$. Ressalta-se que esse resultado foi ratificado pelo teste estatístico de Kendall $\left(\chi_{2 ; 0,05}^{2}=2,83 ; p=0,24\right)$. Sendo assim, concluiu-se que, de maneira geral, os enfermeiros apresentavam concordância na identificação dos diagnósticos de enfermagem nesse período.

Nos períodos trans e pós-operatório imediato, o teste estatístico de Friedman mostrou a existência de pelo menos uma diferença estatisticamente significante entre os totais de postos para a freqüência dos três enfermeiros $\left(\chi_{2 ; 0,05}^{2}=15,573, p \cong 0 ; \chi_{2 ; 0,05}^{2}=15,24 ; p \cong 0\right.$ respectivamente $)$. Esse resultado também foi ratificado pelo teste estatístico de Kendall $\left(\chi_{2: 0.05}^{2}=13,34, p=0,001 ; \chi_{2: 0.05}^{2}=16,19, p \cong 0\right.$ respectivamente).

Mediante a aplicação dos testes de comparações múltiplas, observou-se diferença estatisticamente significante entre o total de postos para frequiência do enfermeiro $1 \mathrm{e}$ os totais de postos dos demais enfermeiros $(p=0,001 \mathrm{e} p=0,0028$ nas comparações entre enfermeiros 1 e 2 e enfermeiros 1 e 3 , respectivamente). Vale ressaltar que o enfermeiro 1 apresentou total de postos de frequiência inferior aos outros enfermeiros. Diante desses resultados, observamos maior homogeneidade entre os enfermeiros 2 e 3 e, portanto, optamos por excluir o enfermeiro 1 , uma vez que não seria possível testar a concordância ou não com LEG no estabelecimento dos diagnósticos, com enfermeiros que apresentavam diferenças estatísticas significantes entre si. Desse modo, somente as categorias diagnósticas identificadas pelos enfermeiros 2 e 3 foram submetidas às análises estatísticas posteriores.
Ressaltamos novamente que estes testes foram realizados antes de qualquer teste de concordância com os diagnósticos identificados por LEG.

Vale destacar também que este estudo não teve a pretensão de verificar quais os diagnósticos seriam mais acurados ou ainda quem possuiria maior acurácia na identificação dos diagnósticos, mas sim de verificar a existência ou não de concordância entre LEG e os enfermeiros convidados a participar do estudo.

A seguir foi realizado o teste estatístico Kappa para testar a concordância ou discordância entre os dois enfermeiros e LEG. Este teste foi aplicado apenas nas categorias diagnósticas identificadas por LEG, com freqüência superior ou igual a $50 \%$, ou por pelo menos um dos enfermeiros em pelo menos um dos pacientes.

Dessa forma, para testar a concordância entre as respostas dos dois enfermeiros e LEG, foi elaborado uma tabela de contingência $2 \times 2$ e calculado o coeficiente Kappa, mediante o programa estatístico Statical Package for Social Science (SPSS).

A análise estatística possibilitou a identificação de duas possibilidades:

- discordância entre os enfermeiros - conseqüentemente esta conclusão estatística não permite avaliar o grau de concordância com LEG;

- concordância entre os enfermeiros - esta conclusão estatística se subdivide em duas outras possibilidades: concordância entre os enfermeiros e com LEG e concordância entre os enfermeiros e discordância com LEG.

A seguir, serão apresentados os dados referentes ao grau de concordância e discordância entre os diagnósticos identificados pelos enfermeiros 2 e 3 e LEG, separados pelos períodos: pré, trans e pós-operatório.

\section{RESULTADOS E DISCUSSÃO}

Foram avaliados dezessete pacientes, sendo nove homens e oito mulheres, com idade média de 58 anos. Os procedimentos cirúrgicos realizados foram: 12 (70\%) revascularizações do miocárdio, duas (12\%) trocas de valva mitral, uma $(6 \%)$ plastia de valva mitral e tricúspide, uma (6\%) troca de valva mitral e tricúspide e uma (6\%) troca de valva mitral e plastia de tricúspide. 


\section{Período pré-operatório}

Tabela 1 - Categorias diagnósticas identificadas por LEG noperíodo pré-operatório, comfreqüência superior a $50 \%$ com valores da estatística k e respectivos níveis de significância, e grau de concordância com os enfermeiros - janeiro/maio de 2001

\begin{tabular}{|c|c|c|c|}
\hline Categoria diagnóstica & $\begin{array}{l}\text { Freqûencia(\%) } \\
\text { (LEG) }\end{array}$ & $\mathrm{K}$ & $\mathrm{p}$ \\
\hline I ntolerância à atividade & 88,2 & 1,0 & 0,0 \\
\hline $\begin{array}{l}\text { Risco para disfunção } \\
\text { neurovascular periférica }\end{array}$ & 82,4 & 0,11 & 0,33 \\
\hline Risco para infecção & 76,5 & 1,0 & 0,0 \\
\hline Déficit de conhecimento & 76,5 & 0,27 & 0,21 \\
\hline Padrão respiratório ineficaz & 70,6 & 0,20 & 0,32 \\
\hline $\begin{array}{l}\text { Perfusão tissular } \\
\text { cardiopulmonar alterada }\end{array}$ & 70,6 & $*$ & 0,07 \\
\hline Dor & 70,6 & 0,08 & 0,62 \\
\hline $\begin{array}{l}\text { Padrões de sexualidade } \\
\text { alterados }\end{array}$ & 58,8 & 0,08 & 0,34 \\
\hline Distúrbios no padrão do sono & 52,9 & 0,39 & 0,04 \\
\hline
\end{tabular}

Nota: * as respostas referentes a essa categoria diagnóstica não permitiram identificar o coeficiente de Kappa, sendo realizado o teste binomial.

Observa-se na Tabela 1 que as categorias diagnósticas Intolerância à atividade, Risco para infecção e Distúrbio no padrão do sono apresentaram concordância entre os enfermeiros e com LEG.

A categoria diagnóstica Intolerância à atividade apresentou concordância absoluta com os outros enfermeiros $(\mathrm{K}=1,0 ; \mathrm{p}=0)$. Sabe-se que pacientes com insuficiência cardíaca possuem intolerância à atividade física e podem apresentar dificuldades para deambular, mesmo que por um curto período, devido ao desconforto respiratório, fadiga e palpitação que essa atividade acarreta. Além da incapacidade do coração em manter um débito cardíaco suficiente para satisfazer as necessidades teciduais de oxigênio e a redução do fluxo sanguíneo periférico, alguns estudos comprovaram que estes pacientes apresentam também alterações morfológicas e metabólicas na musculatura esquelética, interferindo na capacidade desses indivíduos em realizar determinadas atividades ${ }^{(20-21)}$.

Na identificação da categoria diagnóstica Risco para infecção também observou-se concordância absoluta $(\mathrm{k}=1,0$ $\mathrm{e} p=0$ ) com os outros enfermeiros, validando a presença dessa categoria diagnóstica nestes indivíduos.

Houve também concordância com os outros enfermeiros na identificação da categoria diagnóstica Distúrbio no padrão do sono $(\mathrm{k}=0,39 ; \mathrm{p}=0,04)$. Esse resultado pode ser justificado pelo fato do hospital, com seus sons, odores e visões peculiares ser considerado estranho e até assustador para o paciente que dele se utiliza, gerando ansiedade e medo. Esse medo pode ter como resultado aumento da tensão muscular, do estado de alerta e da percepção e, conse- qüentemente, a impossibilidade de repousar e dormir ${ }^{(22)}$. Além disso, existem os fatores fisiopatológicos resultantes da insuficiência cardíaca que interferem diretamente na capacidade de indivíduo de iniciar ou manter o sono como, por exemplo, a nictúria, a terapia medicamentosa (diuréticos), a ortopnéia e a dispnéia paroxística noturna.

No caso da categoria diagnóstica Perfusão tissular cardiopulmonar alterada, a estatística de Kappa não pode ser obtida pelo fato de as respostas de um dos enfermeiros serem constantes, não permitindo a construção de uma tabela de contingência $2 \times 2$. Desse modo, para essa categoria realizou-se o Teste binomial $(\mathrm{p}=0,07)$ que indicou não existir evidência estatística de concordância. Sabe-se que na doença arterial coronariana, o calibre das artérias coronárias se encontra reduzido devido às lesões ateroscleróticas, que diminuem o suprimento sanguíneo para determinada área do coração e, conseqüentemente, levam a uma Perfusão tissular cardiopulmonar. Para alguns autores essa categoria diagnóstica constitui um problema colaborativo e não um diagnóstico de enfermagem propriamente dito, pois representa uma situação em que a enfermagem não pode atuar independente das ações médicas, ou seja, a enfermagem não pode elaborar um plano de cuidados para "reestabelecer" a perfusão cardiopulmonar do indivíduo ${ }^{(23)}$.

De acordo com a Tabela 1, observa-se que para as categorias diagnósticas Risco para disfunção neurovascular periférica, Déficit de conhecimento, Padrão respiratório ineficaz, Dor e Padrões de sexualidade alterados não houve evidências estatísticas de concordância entre os enfermeiros e LEG.

\section{Período trans-operatório}

Tabela 2 - Categorias diagnósticas identificadas por LEG no período trans-operatório, com freqüência superior a $50 \%$ com valores da estatística $k$ e respectivos níveis designificância, egrau deconcordância com os enfermeiros - janeiro/maio de 2001

\begin{tabular}{lccc}
\hline Categoria diagnóstica & $\begin{array}{c}\text { Freqûencia(\%) } \\
\text { (LEG) }\end{array}$ & K & p \\
\hline Risco para infecção & 100 & $\#$ & $\#$ \\
Risco para desequilíbrio no & 100 & $\#$ & $\#$ \\
volume de líquidos & 100 & $\#$ & $\#$ \\
Risco para aspiração & 100 & $\#$ & $\#$ \\
Proteção alterada & 100 & $\#$ & $\#$ \\
Integridade da pele prejudicada & 100 & $\#$ & $\#$ \\
Risco para disfunção & & & \\
neurovascular periférica & 100 & $\#$ & $\#$ \\
Risco para lesão perioperatória & & & \\
de posicionamento & 100 & $*$ & 0 \\
Troca de gases prejudicada & 94,1 & 0,45 & 0,03 \\
Risco para temperatura & & & \\
corporal alterada & & &
\end{tabular}

Nota: \#categorias diagnósticas que apresentaram concordância total independente de análise estatística; * as respostas referentes a essa categoria diagnóstica não permitiram identificar o coeficiente de Kappa, sendo realizado o teste binomial. 
Observa-se na Tabela 2 que das categorias diagnósticas identificadas por LEG com freqüência superior a $50 \%$ no período trans-operatório, sete apresentaram concordância total, isto é, $100 \%$ de concordância entre os enfermeiros e LEG.

Este fato, talvez seja resultante do intenso controle que $\mathrm{o}$ anestesista, o perfusionista (profissional responsável pela circulação extracorpórea - CEC) e o cirurgião têm dos mecanismos fisiológicos do paciente no período intra-operatório. O paciente, nesse período, é submetido a uma série de "situações artificiais" como: indução do coma (agentes anestésicos); indução de parada cardíaca (cardioplegia); inibição do sistema da coagulação (heparinização sistêmica); oxigenação e fluxo sanguíneos artificiais (CEC) e indução da hipotermia, podendo acarretar problemas similares a todos os pacientes.

A categoria Risco para temperatura corporal alterada apresentou concordância entre os enfermeiros, pois ambos não identificaram tal categoria diagnóstica, e discordância com a pesquisadora $(\mathrm{K}=0,45 ; \mathrm{p}=0,03)$. A justificativa para essa não concordância com a pesquisadora deve-se ao fato dos enfermeiros estabelecerem a Termorregulação ineficaz para indicar problemas relacionados a manutenção da temperatura corporal dentro dos parâmetros aceitáveis. Neste caso, o problema da não concordância clínica está na interpretação da categoria diagnóstica.

A NANDA define Termorregulação ineficaz como estado no qual a temperatura do indivíduo flutua entre a hipotermia e a hipertermia ${ }^{(18)}$. De fato, o paciente apresenta a constante oscilação de temperatura durante a cirurgia cardíaca, no entanto essas oscilações ocorrem por indução. Normalmente, o paciente chega a sala cirúrgica com temperatura orofaríngea variando entre $36^{\circ}$ a $35^{\circ} \mathrm{C}$, no início da CEC é induzido a ficar hipotérmico e ao final da cirurgia é reaquecido. Essas oscilações de temperatura, embora induzidas, tornam difícil definir se o paciente apresenta Hipotermia, Termorregulação ineficaz ou Risco para temperatura corporal alterada. Considerando as peculiaridades da cirurgia cardíaca (resfriamento no início da CEC e reaquecimento ao final e prolongado tempo cirúrgico) e os fatores intrínsecos do paciente (obesidade e idade), o Risco para temperatura corporal alterada, definido como estado no qual um indivíduo corre o risco de não conseguir manter a temperatura corporal dentro dos parâmetros normais $^{(18)}$, representa a categoria diagnóstica que melhor se aplica a esse período.

Conforme apresentado na Tabela 2 pode-se observar que houve discordância entre os enfermeiros com LEG na categoria Troca de gases prejudicada.

\section{Periodo pós-operatório imediato}

Tabela 3 - Categorias diagnósticas identificadas por LEG no período pós-operatório imediato, com freqüência superior a $50 \%$ com valores da estatística $k e$ respectivos níveis de significância, e grau de concordância com os enfermeiros - janei ro/maio de 2001.

\begin{tabular}{|c|c|c|c|}
\hline Categoria diagnóstica & $\begin{array}{l}\text { Freqûencia(\%) } \\
\text { (LE G) }\end{array}$ & K & $p$ \\
\hline Risco para infecção & 100 & \# & \# \\
\hline $\begin{array}{l}\text { Risco para disfunção } \\
\text { neurovascular periférica }\end{array}$ & 100 & \# & \# \\
\hline $\begin{array}{l}\text { Risco para lesão perioperatória } \\
\text { de posicionamento }\end{array}$ & 100 & \# & \# \\
\hline Mobilidade física prejudicada & 100 & \# & \# \\
\hline $\begin{array}{l}\text { Déficit no autocuidado para } \\
\text { banho-higiene }\end{array}$ & 100 & \# & \# \\
\hline Risco para aspiração & 100 & \# & \# \\
\hline Proteção alterada & 100 & \# & \# \\
\hline I ntegridade da pele prejudicada & 100 & \# & \# \\
\hline $\begin{array}{l}\text { Risco para desequilíbrio no } \\
\text { volume de líquidos }\end{array}$ & 100 & 0,19 & 0,40 \\
\hline $\begin{array}{l}\text { Alterações sensoriais } \\
\text { de percepção }\end{array}$ & 100 & $\#$ & \# \\
\hline Comunicação verbal prejudicada & 94,1 & 1,0 & 0 \\
\hline $\begin{array}{l}\text { Risco para temperatura } \\
\text { corporal alterada }\end{array}$ & 94,1 & $-0,13$ & 0 \\
\hline $\begin{array}{l}\text { Desobstrução ineficaz das vias } \\
\text { aéreas }\end{array}$ & 82,3 & 1,00 & 0 \\
\hline Troca de gases prejudicada & 64,7 & $-0,65$ & 0 \\
\hline Dor & 52,9 & 0,46 & 0,05 \\
\hline
\end{tabular}

Nota: \# categorias diagnósticas que apresentaram concordância ou discordância total independente de análise estatística.

$\mathrm{Na}$ análise da Tabela 3 observa-se que as categorias diagnósticas Risco para infecção, Risco para disfunção neurovascular periférica, Risco para lesão perioperatória de posicionamento, Mobilidade física prejudicada, Risco para aspiração, Proteção alterada, Integridade da pele prejudicada, Alterações sensoriais/de percepção, Comunicação verbal prejudicada, Desobstrução ineficaz das vias aéreas e Dor) apresentaram concordância com os enfermeiros.

O Risco para infecção, identificado em todos os pacientes tanto por LEG como pelos enfermeiros (concordância total), está associado, entre outros, aos fatores relacionados ao tratamento (cirurgia, presença de vias invasivas, terapia medicamentosa). Além dos procedimentos invasivos e da defesa primária insuficiente, provocada pelo trauma cirúrgico, vários outros fatores influenciam a incidência de infecção na ferida operatória, entre eles as condições clínicas pré-operatórias do paciente, as condições técnicas em que a cirurgia foi realizada e a permanência hospitalar préoperatória ${ }^{(24)}$.

Associada ao Risco para infecção está à categoria diagnóstica Proteção alterada, definida como estado no qual um indivíduo experimenta uma diminuição na capacidade de proteger-se contra ameaças internas ou externas, como doenças ou lesões ${ }^{(18)}$, e que também apresentou concordância total entre LEG e os enfermeiros. 
O paciente submetido a cirurgia cardíaca apresenta Proteção alterada devido aos vários procedimentos inerentes a cirurgia (invasão de estruturas no corpo, punções etc), mas em especial devido a CEC. Ao contrário da maioria dos procedimentos invasivos, a CEC produz significativas alterações no equilíbrio fisiológico do organismo, constituindo-se num agente agressor complexo e multifatorial. Dentre as alterações produzidas pela CEC estão à inibição do sistema de coagulação e o contato do sangue com superfícies estranhas, isto é, não endoteliais, como os oxigenadores e circuitos $^{(25)}$.

Diante do exposto, observamos que no período transoperatório de cirurgia cardíaca o paciente apresenta Proteção alterada, devido às alterações no sistema de coagulação e nas células sanguíneas, e conseqüentemente, Risco para infecção. Desse modo, não se faz necessário estabelecer as duas categorias diagnósticas em discussão, uma vez que a prescrição de enfermagem realizada para a Proteção alterada seria a mesma que para o Risco para infecção.

Os resultados obtidos neste estudo confirmam os obtidos por outros pesquisadores que também identificaram as seguintes categorias diagnósticas Proteção alterada, Risco para infecção, Integridade da pele prejudicada, Risco para lesão perioperatório de posicionamento e Risco para disfunção neurovascular periférica no período perioperatório $^{(26)}$.

A categoria diagnóstica Déficit no autocuidado para banho/higiene apresentou discordância total, isto é, foi identificada em todos os pacientes pela pesquisadora, mas em nenhum pelos dois enfermeiros.

De acordo com a Tabela 3 observa-se que para a categoria diagnóstica Risco para desequilíbrio no volume de líquidos não houve evidências estatísticas de concordância. Observa-se também que houve discordância entre os enfermeiros com LEG no estabelecimento das seguintes categorias diagnósticas: Risco para temperatura corporal alterada e Troca de gases prejudicada.

Não se esperava obter concordância em todas as categorias diagnósticas identificadas por LEG, pois sabe-se que existem diferenças individuais como, por exemplo, a experiência profissional e o conhecimento que interferem na capacidade de interpretar com precisão os dados e, conseqüentemente, de estabelecer um diagnóstico.

\section{CONCLUSÕESE CONSIDERAÇÕES FINAIS}

No período pré-operatório as categorias diagnósticas Intolerância à atividade, Risco para infecção e o Distúrbio no padrão do sono apresentaram concordância com os enfermeiros e LEG. Nas categorias Risco para disfunção neurovascular periférica, Déficit de conhecimento, Padrão respiratório ineficaz, Dor e Padrões de sexualidade alterados não houve evidências estatísticas de concordância entre os enfermeiros e com LEG.

No período trans-operatório, das nove categorias diagnósticas identificadas por LEG com freqüência superior a $50 \%$, as categorias Risco para infecção, Risco para desequilíbrio no volume de líquidos, Risco para aspiração, Proteção alterada, Integridade da pele prejudicada, Risco para disfunção neurovascular periférica e Risco para lesão perioperatória de posicionamento apresentaram $100 \%$ de concordância entre os enfermeiros e LEG. A categoria Risco para temperatura corporal alterada apresentou concordância entre os enfermeiros, pois ambos não identificaram tal categoria diagnóstica, e discordância com LEG. Nesse período houve discordância entre os enfermeiros com LEG na categoria Troca de gases prejudicada.

No período pós-operatório as categorias diagnósticas Risco para infecção, Risco para disfunção neurovascular periférica, Risco para lesão perioperatória de posicionamento, Mobilidade física prejudicada, Risco para aspiração, Proteção alterada, Integridade da pele prejudicada, Alterações sensoriais/de percepção, Comunicação verbal prejudicada, Desobstrução ineficaz das vias aéreas e Dor apresentaram concordância total com os enfermeiros. A categoria diagnóstica Déficit no autocuidado para banho/higie$n e$ apresentou discordância total, isto é, foi identificada em todos os pacientes por LEG, mas em nenhum pelos dois enfermeiros. Também houve discordância no estabelecimento das categorias diagnósticas Risco para temperatura corporal alterada e Troca de gases prejudicada. A categoria diagnóstica Risco para desequilíbrio no volume de líquidos não houve evidências estatísticas de concordância.

Este estudo fornece uma base para a implementação do processo de enfermagem no período perioperatório de cirurgia cardíaca. A identificação dos diagnósticos de enfermagem nesse período poderá auxiliar os enfermeiros na elaboração de intervenções fundamentadas e adequadas às necessidades individuais de cada paciente, colaborando para a implementação de ações rápidas e eficazes para a resolução dos problemas identificados. 
(1) Zipes DP, Wellens HJ. Sudden cardiac death. Circulation. 1998;98(21):2334-51.

(2) Zheng ZJ, Croft JB, Giles WH, Mensah GA. Sudden cardiac death in United States, 1989 to 1998. Circulation. 2001;104(18):2158-63.

(3) Black JM, Matassarin-Jacobs E. Enfermagem médico-cirúrgica: uma abordagem psicofisiológica. $4^{\mathrm{a}}$ ed. Rio de Janeiro: Guanabara Koogan; 1996.

(4) Iglésias JCR, Oliveira Júnior JL, Dallan LAO, Lourenção Júnior A, Stolf NAG. Preditores de mortalidade hospitalar no paciente idoso portador de doença arterial coronária. Rev Bras Cir Cardiovasc. 2001;12:94-104.

(5) Kim MJ, Amoroso-Seritella R, Gulanick M, Moyer K. Clinical validation of cardiovascular nursing diagnoses. In: Kim MJ, Mcfarland GK, Mclane AM, editors. Classification of nursing diagnoses: proceedings of the fifth national conferences. Saint Louis: Mosby; 1984. p. 128.

(6) Faria MFG. Diagnósticos de enfermagem respiratórios em pacientes cardíacos cirúrgicos [dissertação]. Ribeirão Preto: Escola de Enfermagem de Ribeirão Preto/USP; 2000.

(7) De Gasperis M. Implementing nursing diagnosis in the critical care setting. Dimens Crit Care Nurs. 1983;2(1):44-9.

(8) Kuhn RCE. American Association of Critical-Care Nurses. In: Proceeedings of $9^{\text {th }}$ NANDA Conference on Classification of Nursing Diagnoses; 1990 mar. 17-19, Orlando. Orlando: NANDA; 1990. p. 209.

(9) Rantz ME. Integrating nursing diagnosis in long-term care. In: Proceedings of $11^{\text {th }}$ NANDA Conference on Classification of Nursing Diagnoses; 1994; mar. 26-30; Nashville. Nashville: NANDA; 1994. p. 29.

(10) Freitas MC. Trabalhando com diagnóstico de enfermagem no estágio de unidade de terapia intensiva. In: Anais do $1^{\circ} \mathrm{Simpósio}$ Nacional sobre Diagnósticos de Enfermagem e do $3^{\circ}$ Encontro Cearense sobre Diagnósticos de Enfermagem; 1996; Fortaleza. Fortaleza: ABEn-Seção-CE; 1996. p. 8.

(11) Pasini D, Alvim I, Kanda L, Mendes RSP, Cruz DALM. Diagnósticos de enfermagem de pacientes em unidades de terapia intensiva. In: Anais do $1^{\circ}$ Simpósio Nacional sobre Diagnósticos de Enfermagem e do $3^{\circ}$ Encontro Cearense sobre Diagnósticos de Enfermagem; nov. 25-27 1996; Fortaleza. Fortaleza: ABEn-Seção-CE; 1996. p. 59.

(12) Sackett DL, Haynes RB, Tugwell P, Guyatt GH, editors. Clinical epidemiology: a basic science for clinical medicine. $2^{\text {nd }}$ ed. Boston: Lippincott Willians \& Wilkins; 1991.

(13) Cavalcanti AB, Avezum A, Sousa AG, Rassi Júnior A, Coutinho M, Mesquita ET, et al. Semiologia médica: a contribuição da cardiologia baseada em evidências. Rev Soc Cardiol Est São Paulo. 2001;11:176-88.
(14) Voytovich AE, Rippey RM, Suffredini A. Premature conclusions in diagnostic reasoning. J Med Educ. 1985;60(4):302-7.

(15) Maslow AH. Motivation and personality. $2^{\text {nd }}$ ed. New York: Harper \& Row; 1970.

(16) Mohana J. O mundo e eu. $2^{a}$ ed. Rio de Janeiro: Agir; 1964.

(17) Risner PB. Diagnosis: analysis and synthesis of data. In: Christensen PJ, Kenney JW, editors. Nursing process: conceptual models. $4^{\text {th }}$ ed. Saint Louis: Mosby; 1990. p. 132 57.

(18) North American Nursing Diagnosis Association (NANDA). Nursing diagnosis: definitions \& classification. Saint Louis: NANDA; 2000.

(19) Siegel S. Estatística não-paramétrica. São Paulo: McGrawHill; 1975.

(20) Drexler H, Riede U, Munzel T, Konig H, Funke E, Just H. Alterations of skeletal muscle in chronic heart failure. Circulation. 1992; 85(5):1751-9.

(21) Minotti JR, Christoph I, Oka R, Weiner MW, Wells L, Massie BM. Impaired skeletal muscle function in patients with congestive heart failure. Relationship to systemic exercise performance. J Clin Invest 1991; 88(6):2077-82.

(22) Atkinson LD, Murray ME. Fundamentos de enfermagem: introdução ao processo de enfermagem. Rio de Janeiro: Guanabara Koogan; 1989.

(23) Carpenito LJ. Diagnósticos de enfermagem: aplicação à prática clínica. $6^{a}$ ed. Porto Alegre: Artes Médicas; 1997.

(24) Rabhae GN, Ribeiro Filho N, Fernandes AT. Infecção do sítio cirúrgico. In: Fernandes AT, Fernandes MOVF, editores. Infecção hospitalar e suas interfaces na área de saúde. São Paulo: Atheneu; 2000. p. 479-502.

(25) Souza MHL, Elias DO. Resposta inflamatória sistêmica à circulação extracorpórea. In: Souza MHL, Elias DO, editores. Fundamentos da circulação extracorpórea; Rio de Janeiro: Alfa Rio; 1999. p. 374-91.

(26) Killen AR, Kleinbeck SV, Gollar K, Schuchardt JT, Uebele J. The prevalence of perioperative nurse clinical judgments. AORN J. 1997;65(1):101-8. 